\title{
Current Distribution in Fused Electrical Networks
}

\author{
J.S. Espinoza Ortiz ${ }^{1}$ and Gemunu H. Gunaratne ${ }^{1,2}$ \\ ${ }^{1}$ Department of Physics, University of Houston, Houston, TX77204 \\ ${ }^{2}$ The Institute of Fundamental Studies, Kandy 20000, Sri Lanka
}

Received on 27 December, 2002

\begin{abstract}
Electrical networks provide a natural model to study transport processes such us dielectric breakdown and metal insulator transition in disordered inhomogeneous conductors. We present a calculation for current distribution on an infinite hyper-cubic network due to an external applied current. This is used to compute, analytically, the redistribution of current due to variations in the conductance of a finite number of elements of the network.
\end{abstract}

\section{Introduction}

Electrical network models can be used to study transport phenomena in disordered systems. Examples of such systems include compacted mixtures of conducting and nonconducting materials or homogeneous multi-phase system with varying conductivity. Applications to disordered networks cover dielectric breakdown $[1,2,3]$, metal-insulator transitions [4], and brittle fracture in disordered solids [5, 6, $7,8]$. In particular,such models have provided insights on critical phenomena [9], scale-invariant disorder [10, 9, 3], and size-dependence of the system [11]. In this paper we introduce an exact analytical technique to compute current distributions on model networks.

In the "Effective Medium Theory", the distribution of current in a random network of conductances to which an external current has been applied along a fixed direction must be regarded as due to both an "external field" which increases the mean current, and a "fluctuating local field" due to deviations from a uniform medium. The "mean current" is chosen so that the average of these fluctuation vanish.

We consider fused-conducting networks, where the breakdown currents of an element is assumed to be proportional to its conductance; i.e., breakdown of any conductor occurs when the potential difference across it reaches a fixed pre-set value. In the simplest case, conductances along each axis of a hyper-cube are set equal. In Section II, we calculate how an external current introduced at a node and removed from an adjacent node is distributed on the network [12,13]. The Green's Function derived for this case can be used to calculate the current distribution due to an external field on a network from which a finite number of conductances are removed. Several examples are shown in Section III, for two and three dimensional networks. Subsequently, in section $\mathrm{IV}$, this method is generalized to consider networks where conductances are reduced (as opposed to vanishing) beyond a critical current. Section V presents discussions and conclusions.

\section{Green's Functions for Hyper-cubic Network}

Lets us consider an infinite $d$-dimensional hyper-cubic network where all conductances along the $m^{t h}$ direction $\left(\hat{\mathbf{u}}_{\mathbf{m}}\right)$ are assumed to be equal to $\sigma_{m}(m=1,2, \ldots, d)$. In this section we calculate the distribution of current on this network due to a unit external current introduced at the origin and removed from an adjacent node, say $\mathbf{a}$. This result was obtained by using a Green's function method by Kirkpatric $[12,11]$ and is reproduced here for completeness.

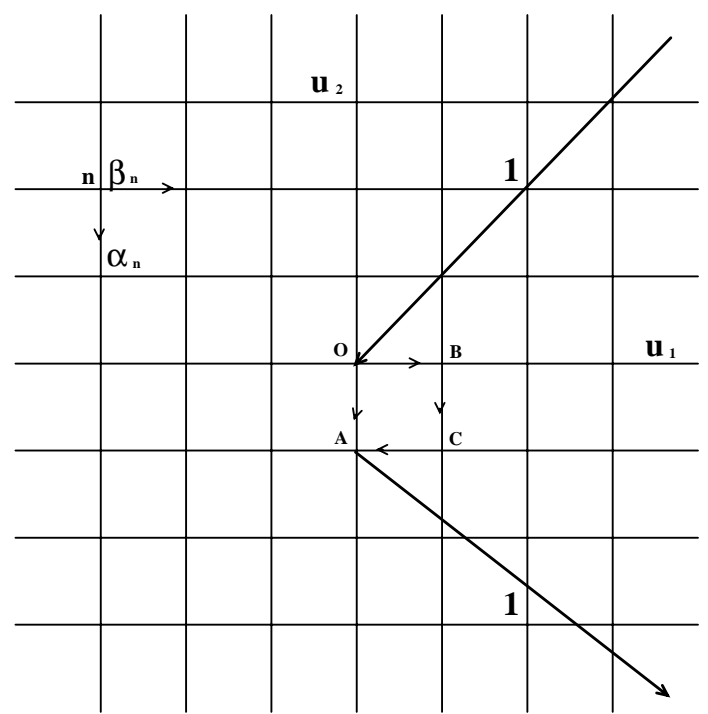

Figure 1. Scheme to compute the current distribution due to a unit external current introduced at the origin and removed from an adjacent node.

Denote the potential at the node $\mathbf{n}=\left(n_{1}, n_{2}, \ldots, n_{d}\right)$, by $V(\mathbf{n})$ and the current on the conductor joining nodes $\mathbf{n}$ and $\mathbf{n}+\hat{\mathbf{u}}_{\mathbf{m}}$ by $\mathbf{J}_{m}(\mathbf{n})$; then,

$$
\mathbf{J}_{m}(\mathbf{n})=\sigma_{m}\left[V(\mathbf{n})-V\left(\mathbf{n}+\hat{\mathbf{u}}_{\mathbf{m}}\right)\right]
$$


The potentials $V(\mathbf{n})$ can be obtained using the Kirchhoff's rule at each node; i.e.,

$$
\begin{array}{ccc}
\sum_{m=1}^{d} & \sigma_{m} & \left\{\left[V(\mathbf{n})-V\left(\mathbf{n}+\hat{\mathbf{u}}_{\mathbf{m}}\right)\right]\right. \\
& + & \left.\left[V(\mathbf{n})-V\left(\mathbf{n}-\hat{\mathbf{u}}_{\mathbf{m}}\right)\right]\right\}=\left(\delta_{\mathbf{n}, \mathbf{0}}-\delta_{\mathbf{n}, \mathbf{a}}\right)
\end{array}
$$

where the term in the right side is the externally applied current. Eqn. (2) is easily solved by using the Fourier transform $\hat{V}(\mathbf{k})=\sum_{\mathbf{n}} e^{-i \mathbf{n} \mathbf{k}} V(\mathbf{n})$, which satisfies

$$
\sum_{m=1}^{d} 2 \sigma_{m}\left[1-\cos \left(\hat{\mathbf{u}}_{\mathbf{m}} \cdot \mathbf{k}\right)\right] \hat{V}(\mathbf{k})=\left(1-e^{-i \mathbf{a} \mathbf{k}}\right)
$$

Thus

$$
\hat{V}(\mathbf{k})=\frac{\left(1-e^{-i \mathbf{a k}}\right)}{\sum_{m=1}^{d} 2 \sigma_{m}\left[1-\cos \left(k_{m}\right)\right]},
$$

where $k_{m}=\hat{\mathbf{u}}_{\mathbf{m}} \cdot \mathbf{k}$. Hence

$$
V(\mathbf{n})=\frac{1 / 2}{(2 \pi)^{d}} \int_{-\pi}^{\pi} d \mathbf{k} e^{i \mathbf{n} \mathbf{k}} \hat{V}(\mathbf{k})
$$

and

$$
\mathbf{J}_{m}(\mathbf{n})=\frac{\sigma_{m}}{2(2 \pi)^{d}} \int_{-\pi}^{\pi} e^{i \mathbf{n k}} \frac{\left[1-e^{-i \mathbf{k}_{\mathbf{a}}}-e^{i k_{m}}+e^{i\left(k_{m}-\mathbf{k}_{\mathbf{a}}\right)}\right]}{\sum_{m /=1}^{d} \sigma_{m \prime}\left[1-\cos \left(k_{m \prime}\right)\right]} d \mathbf{k} .
$$

Next, let us specialize to networks in two dimensions with equal conductances $\left(\sigma_{m}=1\right)$, and define $\mathbf{a}=\hat{\mathbf{u}}_{\mathbf{2}}, \alpha_{(\mathbf{n})} \equiv$ $J_{y}(\mathbf{n})$ and $\beta_{(\mathbf{n})} \equiv J_{x}(\mathbf{n})$, see Fig. 1. In order to obtain expressions for $\alpha^{\prime} s$ and $\beta^{\prime} s$ is also necessary to consider implicit symmetries on the networks; i.e.,

$$
\begin{aligned}
& \alpha_{\left(n_{x}, n_{y}\right)}=\alpha_{\left(n_{x},-n_{y}\right)}=\alpha_{\left(-n_{x}, n_{y}\right)}=\alpha_{\left(-n_{x},-n_{y}\right)}, \\
& \beta_{\left(n_{x}, n_{y}\right)}=-\beta_{\left(n_{x},-n_{y}\right)}=-\beta_{\left(-n_{x}, n_{y}\right)}=\beta_{\left(-n_{x},-n_{y}\right)} .
\end{aligned}
$$

These are easily obtained by taking into account the flux of current in the $O A B C$ cell and by reflecting it with respect to $O A$, see Fig. 1.

Combining Eqns. (5) and (6) gives

$$
\begin{aligned}
& \alpha_{(\mathbf{n})}=\frac{1}{\pi^{2}} \int_{0}^{\pi} \quad \frac{\left[1-\cos \left(k_{y}\right)\right] \cos \left(n_{x} k_{x}\right) \cos \left(n_{y} k_{y}\right)}{2-\cos \left(k_{x}\right)-\cos \left(k_{y}\right)} d \mathbf{k}, \\
& \beta_{(\mathbf{n})}=\frac{2}{\pi^{2}} \int_{0}^{\pi} \frac{\sin \left(k_{x} / 2\right) \sin \left(k_{y} / 2\right) \sin \left[\left(n_{x}+1 / 2\right) k_{x}\right]}{2-\cos \left(k_{x}\right)-\cos \left(k_{y}\right)}
\end{aligned}
$$

This analysis can be easily extended to compute the $\alpha$ 's and $\beta$ 's in three dimensions. These are calculated using configuration analogous to Fig. 1 with the unit external current is introduced along the $\hat{u}_{3}$ direction. Then

$$
\begin{aligned}
\alpha_{(\mathbf{n})}=\frac{1}{\pi^{3}} \int_{0}^{\pi} & \frac{\left[1-\cos \left(k_{z}\right)\right] \cos \left(n_{x} k_{x}\right) \cos \left(n_{y} k_{y}\right) \cos \left(n_{z} k_{z}\right)}{3-\cos \left(k_{x}\right)-\cos \left(k_{y}\right)-\cos \left(k_{z}\right)} d \mathbf{k} \\
\beta_{(\mathbf{n})}^{x}=\frac{2}{\pi^{3}} \int_{0}^{\pi} & \frac{\sin \left(k_{x} / 2\right) \sin \left(k_{z} / 2\right) \sin \left[\left(n_{x}+1 / 2\right) \mathbf{k}\right] \cos \left(n_{y} k_{y}\right)}{3-\cos \left(k_{x}\right)-\cos \left(k_{y}\right)-\cos \left(k_{z}\right)} \\
& \times \sin \left[\left(n_{z}+1 / 2\right) \mathbf{k}\right] d \mathbf{k} \\
\beta_{(\mathbf{n})}^{y}=\frac{2}{\pi^{3}} \int_{0}^{\pi} & \frac{\sin \left(k_{y} / 2\right) \sin \left(k_{z} / 2\right) \cos \left(n_{x} k_{x}\right) \sin \left[\left(n_{y}+1 / 2\right) \mathbf{k}\right]}{3-\cos \left(k_{x}\right)-\cos \left(k_{y}\right)-\cos \left(k_{z}\right)} \\
& \times \sin \left[\left(n_{z}+1 / 2\right) \mathbf{k}\right] d \mathbf{k}
\end{aligned}
$$

Notice, now there are two possible directions for $\beta^{\prime}$ s (i.e. $x$ and $y$, respectively).

Values of $\alpha$ 's and $\beta$ 's given by Eqn. (7-8) for several $\mathbf{n}$ are given in the Appendix, and will be used for computa- tion to follow. In particular $\alpha_{0}=1 / 2$ and $1 / 3$ in two and three dimensions respectively, as can be easily confirmed using arguments based on symmetry and superposition [12] .

The $\alpha$ 's and $\beta$ 's can also be computed perturbatively by 
expanding the denominator as a series in powers of $\cos k_{j}$. Expansion to fourth order gives results that are accurate to within $2 \%$.

\section{Removal of Conductors in Net- works}

In this section we introduce a method to calculate the current distribution on the network when a finite number of conduc-

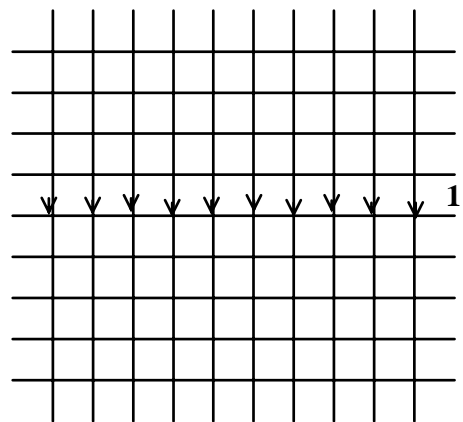

(a)

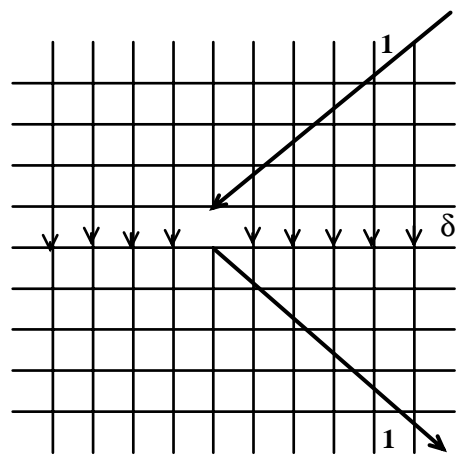

(c) tances are removed.

Consider a two dimensional isotropic $\left(\sigma_{x}=\sigma_{y}=1\right)$ network with an externally applied unit electric field in the $y$-direction. The currents on this configuration are $J_{x}(\mathbf{n})=0, J_{y}(\mathbf{n})=1$, see Fig. 2(a).

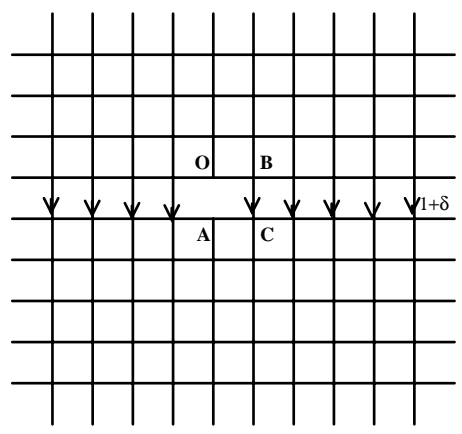

(b)

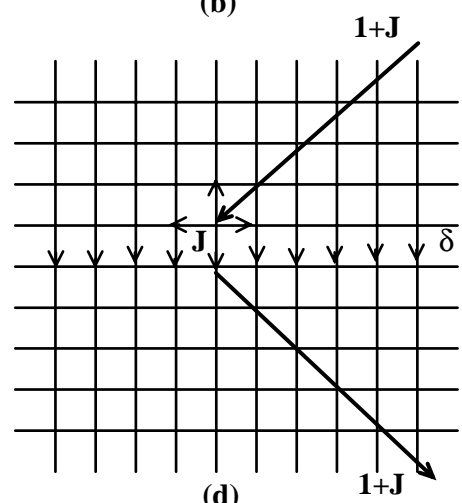

(d)

Figure 2. Scheme to compute the current distribution when a single conductance is removed. In sequence:(a) an isotropic network with a current flow along the $y$-direction. In (b) a conductance is removed, and $\delta$ is the excess of current in a remaining element. Next in (c), a unit external current is introduced in order to compute $\delta$. Finally (d) shows the configuration from which $\delta$ can be evaluated.

First, we enumerate changes in the current distribution due to the removal of the conductor joining the origin to $(0,-1)$, the link $O A$ in Fig. 2(b). The excess current $\delta$ on any remaining element can be considered to be the redistribution of the current that originally passed through the link $O A$, i.e. $(0,0)-(0,-1)$, see Fig. $2(\mathrm{c})$. $\delta$ can be evaluated using specific values of $\alpha$ 's and $\beta$ 's by the following procedure. Consider the complete network, with and external current $(1+J)$ introduced at $(0,0)$ and removed from $(0,-1)$, as shown in Fig. 2(d). $(1+J)$ is chosen so that a current $J$ passes through $(0,0)-(0,-1)$, and the remainder $(=1)$ passes through the rest of the network; i.e., this second part is the solution to the problem illustrated in Fig. 2(c). But from the discussion in Section II, the current passing through $(0,0)-(0,-1)$ is $\alpha_{(0,0)} \times(1+J)$. Hence,

$$
J=\alpha_{(0,0)} \times(1+J) .
$$

Since $\alpha_{(0,0)}=1 / 2$, we find that $J=1$ and that the changes of current in the network are given by,

$$
\begin{aligned}
& \triangle J_{y}(\mathbf{n})=(1+J) \alpha_{\left(n_{x}, n_{y}\right)}=2 \alpha_{\left(n_{x}, n_{y}\right)}, \\
& \triangle J_{x}(\mathbf{n})=(1+J) \beta_{\left(n_{x}, n_{y}\right)}=2 \beta_{\left(n_{x}, n_{y}\right)} .
\end{aligned}
$$

In particular, the current on conductances adjacent to that removed in Fig. 2(b) is

$$
J_{\max }=1+2 \alpha_{(1,0)}=4 / \pi,
$$

as was given by Duxbury et. al. [11]

Finally, we provide results from the analogous calculation for cubic networks of fused conductances. For a single fracture like in Fig. $2, J=1 / 2$ and the value of maxima current for adjacent links are,

$$
\begin{aligned}
J_{\max } & =1+\alpha_{(1,0,0)}(1+J)=1+\alpha_{(0,1,0)}(1+J) \\
& =1+\alpha_{(0,0,1)}(1+J)=1.092625 .
\end{aligned}
$$

Several applications of these formulae are given in Ref. [15] 


\section{Reduction of Conductances}

The next problem we address is how the current distribution of the network changes if the conductance of one bond changes to $\sigma<1$, while the remaining conductors are left unchanged. The current passing thorough this link will decrease causing the remainder to be re-distributed onto the rest of the network. We show how the values of these currents can be calculated.

In Fig. 3(a) we consider the bond $O A$ has its conductance reduced to $\sigma$, and there is a current $I$ passing through it. The remainder $(1-I)$ is redistributed into the network causing an excess of current $\delta$ on remaining elements. This is evaluated using a procedure schematically depicted in Fig. 3(c). Consider the complete network, and an external current $(1-I+J)$ is applied at $O$ and removed from $A$. It is chosen so that a current $J$ passes through the link $O A$ and the remainder $(1-I)$ passes through the rest of the network. Because of this construction, the changes in the current on the cell $O A B C$ (in terms of $\alpha^{\prime}$ s and $\beta^{\prime}$ s) are (as was discussed in Section II),
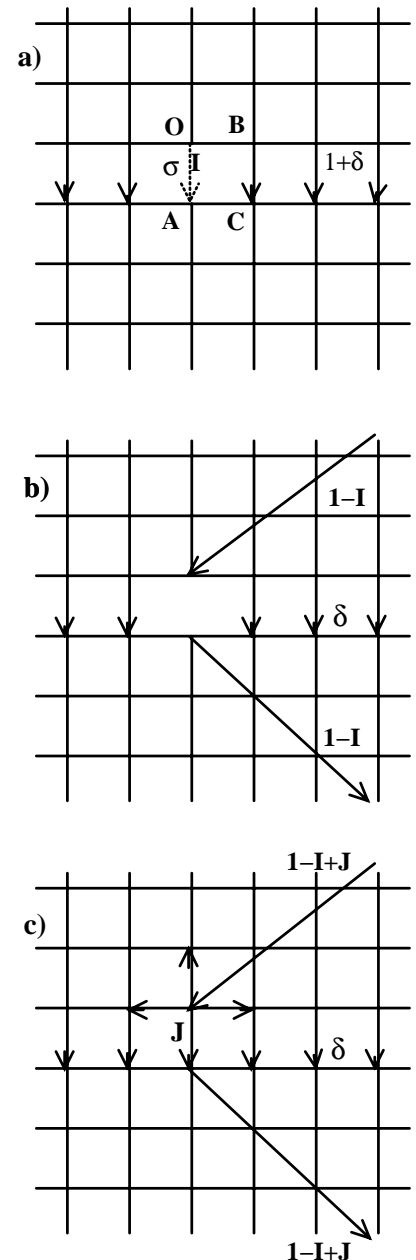

Figure 3. Procedure to compute the current distribution when a central conductance is reduced to $\sigma(<1)$. In (a) the conductance on the link $O A$ is set up to be $\sigma$ and its current is $I$. Then the extra current around its neighborhood is $\delta$. An external current $(1-I)$ is introduced in order to compute $\delta$, in Figure (b). Finally (c) shows the configuration from which $\delta$ is evaluated.

$$
\begin{aligned}
J & =\alpha_{(0,0)}(1-I+J), \\
\triangle J_{x} & =\beta_{(0,0)}(1-I+J), \\
\triangle J_{y} & =\alpha_{(1,0)}(1-I+J), \\
\triangle J_{-x} & =-\beta_{(0,-1)}(1-I+J) .
\end{aligned}
$$

Notice that in order to compute the variations of currents we introduced an auxiliary variable, and the solution require an additional condition. This is obtained by applying Kirckoff's rule into the cell $O A B C$. Thus,

$$
\begin{aligned}
& V_{O A}=V_{O B}+V_{B C}+V_{C A} \\
& I / \sigma=\triangle J_{x}+\left[1+\triangle J_{y}\right]+\triangle J_{-x} .
\end{aligned}
$$

Solving (12) and (13), we find

$$
J=(1-\sigma) /(1+\sigma),
$$

and the changes in currents are:

$$
\begin{aligned}
I & =2 \sigma /(1+\sigma), \\
\triangle J_{x} & =2 \beta_{(0,0)}(1-\sigma) /(1+\sigma), \\
\triangle J_{y} & =2 \alpha_{(1,0)}(1-\sigma) /(1+\sigma), \\
\triangle J_{-x} & =-2 \beta_{(0,0)}(1-\sigma) /(1+\sigma) .
\end{aligned}
$$

Notice that when $\sigma=0$, we retain the results derived in Section III.

a)

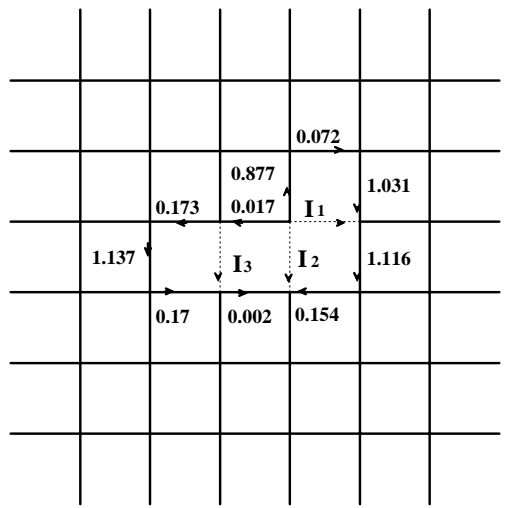

b)

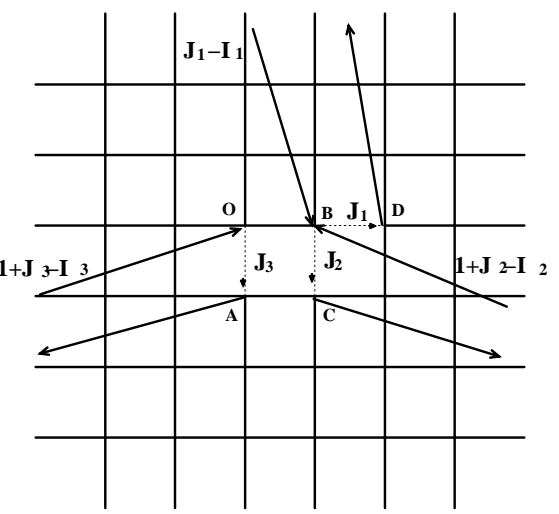

Figure 4. A network with the three conductances (in dashed lines) set up equal to 0.5 , and current distributions on the boundary of this region. The scheme to compute the redistribution of current is shown in (b).

Now we calculate the currents on the network shown in Fig. 4(a). The dashed links (where $\sigma<1$ ) are replaced by externally applied currents as described earlier, as shown in Fig. 4(b). These currents, $J_{1}, J_{2}$ and $J_{3}$ are calculated using, 


$$
\begin{aligned}
& J_{1}=\alpha_{(0,0)}\left(J_{1}-I_{1}\right)+\beta_{(0,0)}\left(1+J_{2}-I_{2}\right)+\beta_{(1,0)}\left(1+J_{3}-I_{3}\right) ; \\
& J_{2}=-\beta_{(-1,0)}\left(J_{1}-I_{1}\right)+\alpha_{(0,0)}\left(1+J_{2}-I_{2}\right)+\alpha_{(1,0)}\left(1+J_{3}-I_{3}\right) ; \\
& J_{3}=-\beta_{(-1,-1)}\left(J_{1}-I_{1}\right)+\alpha_{(-1,0)}\left(1+J_{2}-I_{2}\right)+\alpha_{(0,0)}\left(1+J_{3}-I_{3}\right)
\end{aligned}
$$

and,

$$
\begin{aligned}
I_{1} / \sigma_{1} & =\left[-\beta_{(0,0)}+\alpha_{(1,0)}-\beta_{(0,-1)}\right]\left(J_{1}-I_{1}\right)+\left[-\alpha_{(0,1)}+\beta_{(0,1)}\right. \\
& \left.+\alpha_{(1,1)}\right]\left(1+J_{2}-I_{2}\right)+\left[-\alpha_{(1,1)}+\beta_{(1,1)}+\alpha_{(2,1)}\right]\left(1+J_{3}-I_{3}\right) \\
I_{2} / \sigma_{2} & =\left[-\alpha_{(0,1)}+\alpha_{(-1,1)}\right]\left(J_{1}-I_{1}\right)+\left[-\beta_{(-1,0)}+\beta_{(-1,-1)}\right]\left(1+J_{2}-I_{2}\right)+I_{3} \\
& +\left[-\beta_{(0,0)}+\beta_{(0,-1)}\right]\left(1+J_{3}-I_{3}\right) \\
I_{3} / \sigma_{3} & =\left[-\alpha_{(0,2)}-\beta_{(-1,2)}+\alpha_{(-1,2)}\right]\left(J_{1}-I_{1}\right)+\left[-\beta_{(-2,0)}+\alpha_{(-2,0)}+\beta_{(-2,-1)}\right]\left(1+J_{2}-I_{2}\right) \\
& +1+\left[-\beta_{(-1,0)}+\alpha_{(-1,0)}+\beta_{(-1,-1)}\right]\left(1+J_{3}-I_{3}\right) .
\end{aligned}
$$

The first set of equations are obtained imposing balance of current, and the last ones from applying Kirchoff's laws. For particular case which considers $\sigma_{1}=\sigma_{2}=\sigma_{3}=0.5$, some of the currents on the network are given again in Fig. 4(a) and their values were confirmed through numerical integrations on $100 \times 100$ networks. Values for currents on dashed bounds are: $J_{1}=0.225 ; J_{2}=0.495 ; J_{3}=$ 0.476 , and $I_{1}=0.113 ; I_{2}=0.748 ; I_{3}=0.738$, respectively.

Next let us provide another application considering two clusters interacting, in Fig. 5. Setting up the following values to conductivities: $\sigma_{1}=0.09, \sigma_{2}=0.15, \sigma_{3}=0.6$, and $\sigma_{4}=0.9$, we have solved numerically 8 linear equations to obtain several values of currents which are shown in the same Figure. The respective $J$ 's values were: $J_{1}=$ $0.0287, J_{2}=0.0133, J_{3}=0.2483$, and $J_{4}=0.0767$.

Finally we consider the three dimensional fractured network depicted in Fig. 6. Using analogous arguments above, the currents are computed from:

$$
\begin{aligned}
& J_{1}=\alpha_{(0,0,0)}\left(J_{1}-I_{1}\right)+\beta_{(-1,0,0)}^{y}\left(J_{2}-I_{2}\right)+\beta_{(0,1,-2)}^{y}\left(1+J_{3}-I_{3}\right) ; \\
& J_{2}=\beta_{(0,1,0)}^{x}\left(J_{1}-I_{1}\right)+\alpha_{(0,0,0)}\left(J_{2}-I_{2}\right)+\beta_{(0,1,-1)}^{x}\left(1+J_{3}-I_{3}\right) ; \\
& J_{3}=-\beta_{(0,1,1)}^{y}\left(J_{1}-I_{1}\right)-\beta_{(0,-1,0)}^{x}\left(J_{2}-I_{2}\right)+\alpha_{(0,0,0)}\left(1+J_{3}-I_{3}\right),
\end{aligned}
$$

and

$$
\begin{aligned}
I_{1} / \sigma_{1} & =\left[\beta_{(0,0,0)}^{x}+\alpha_{(1,0,0)}-\beta_{(0,0,-1)}^{x}\right]\left(J_{1}-I_{1}\right)+\left[\alpha_{(-1,0,0)}+\beta_{(-1,0,-1)}^{y}-\alpha_{(-1,1,0)}\right]\left(J_{2}-I_{2}\right) \\
& +\left[\beta_{(0,1,-2)}^{x}+\beta_{(1,1,-2)}^{y}-\beta_{(0,2,-2)}^{x}\right]\left(1+J_{3}-I_{3}\right), \\
I_{2} / \sigma_{2} & =\left[\alpha_{(0,1,0)}+\beta_{(0,1,-1)}^{x}-\alpha_{(1,1,0)}\right]\left(J_{1}-I_{1}\right)+\left[\beta_{(0,0,0)}^{y}+\alpha_{(0,1,0)}\right. \\
& \left.+\beta_{(0,0,-1)}^{y}\right]\left(J_{2}-I_{2}\right)-\left[\beta_{(0,1,-1)}^{y}+\beta_{(0,2,-1)}^{x}-\beta_{(1,1,-1)}^{y}\right]\left(1+J_{3}-I_{3}\right), \\
I_{3} / \sigma_{3} & =\left[\beta_{(0,2,1)}^{x}-\beta_{(1,1,1)}^{y}-\beta_{(0,1,1)}^{x}\right]\left(J_{1}-I_{1}\right)+\left[\alpha_{(1,-1,0)}-\beta_{(0,-1,-1)}^{x}-\alpha_{(0,-1,0)}\right]\left(J_{2}-I_{2}\right) \\
& +\left[\beta_{(0,0,0)}^{x}+\alpha_{(1,0,0)}-\beta_{(0,0,-1)}^{x}\right]\left(1+J_{3}-I_{3}\right)+1 .
\end{aligned}
$$

Setting up conductivities to be $\sigma_{1}=0.5, \sigma_{2}=0.25$ and $\sigma_{3}=0.1$ we have obtained $I_{1}=0.0091, I_{2}=0.0083$ and
$I_{3}=0.1429$, respectively. These conclusions were confirmed numerically. 


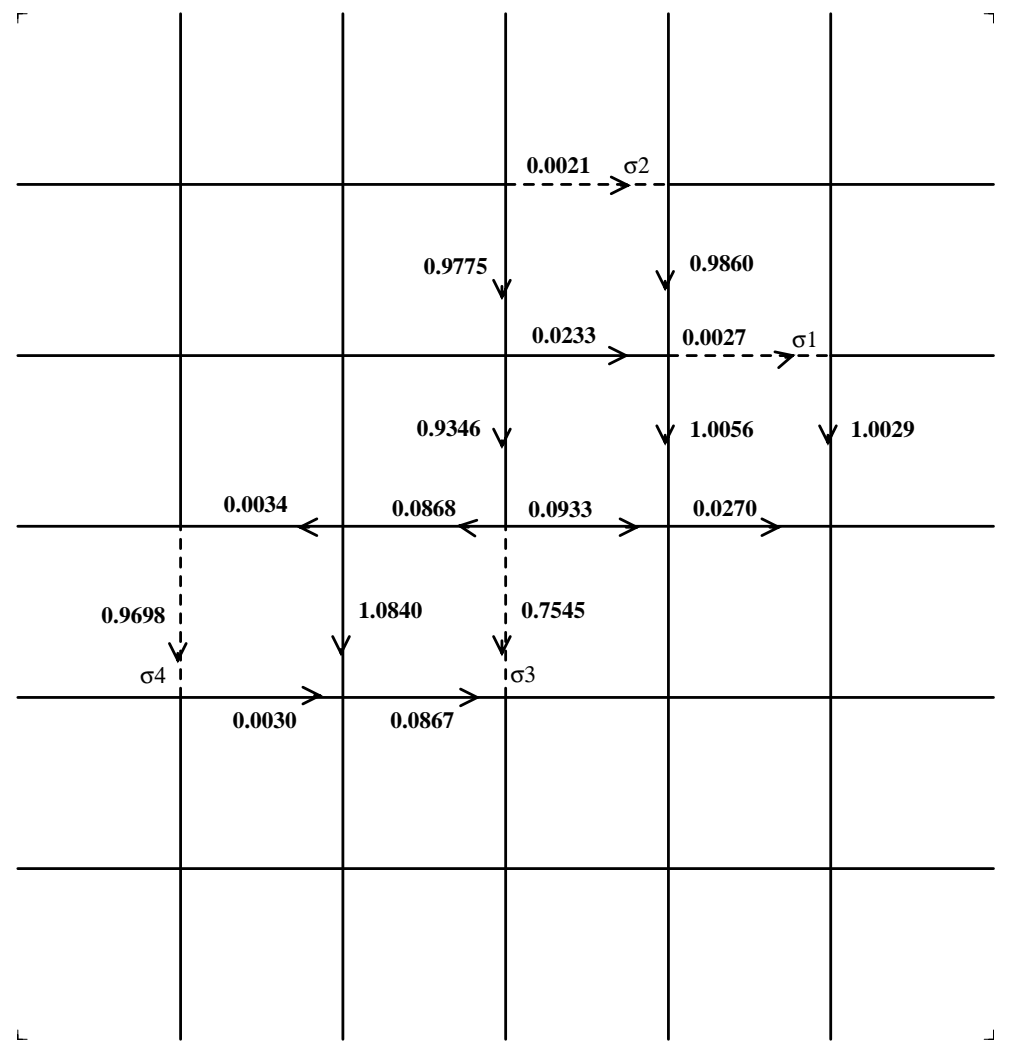

Figure 5. A network with four (reduced) conductances set up to $\sigma_{1}=0.09, \sigma_{2}=0.15, \sigma_{3}=0.6$ and $\sigma_{4}=0.9$ (in dashed lines). Some currents on their neighborhood are also shown.

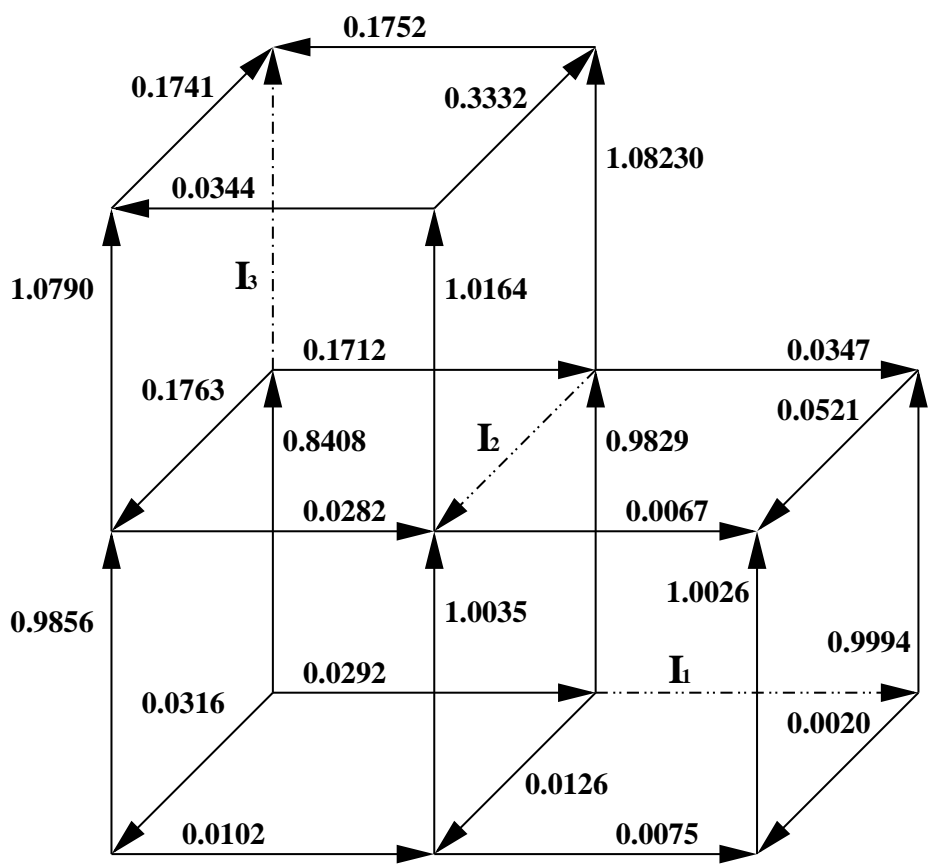

Figure 6. A three-dimensional fractured network with three conductances settled up to $\sigma_{1}=0.5, \sigma_{2}=0.25$ and $\sigma_{3}=0.1$ (in dashed lines). Current distributions on their neighborhood is also shown. 


\section{Discussions and Conclusions}

We have presented a method to compute current distributions on infinite two and three dimensional networks, a finite number of whose conductances differ from unity. The method can be used to calculate (for example) how breakdown strength of a networks reduces due to a "fracture" of $\kappa$ congruent locations whose conductances have "burned". The expression obtained from this calculation can be gen- eralized to elastic networks, one of whose applications is the determination of a relationship between the strength and density of the trabecular bone, with possible applications in using bone strength as a non-invasive diagnostic tool to identify patients with osteoporosis $[15,16]$.

We would like to thank discussions with C. Rajapakse. This work is partially funded by the Office of Naval Research, the National Science Foundation and the ICSC World Laboratory.

\section{Appendix}

Table 1: Values of several $\alpha\left(n_{x}, n_{y}\right)$ 's. In particular $\alpha(0,0)=1 / 2$, and $\alpha(1,0)=1 / 2(4 / \pi-1)$.

\begin{tabular}{ccccccc}
\hline \hline$\frac{n_{y}}{n_{x}}$ & 0 & 1 & 2 & 3 & 4 & 5 \\
\hline \hline 0 & 0.50000 & 0.13662 & 0.04648 & 0.02019 & 0.01080 & 0.00670 \\
1 & 0.13662 & 0.00001 & 0.01455 & 0.01174 & 0.00816 & 0.00571 \\
2 & 0.04648 & 0.01455 & 0.00000 & 0.00404 & 0.00441 & 0.00383 \\
3 & 0.02019 & 0.01174 & 0.00404 & 0.00000 & 0.00162 & 0.00208 \\
4 & 0.01080 & 0.00816 & 0.00441 & 0.00162 & 0.00000 & 0.00080 \\
5 & 0.00670 & 0.00571 & 0.00383 & 0.00208 & 0.00080 & 0.00000 \\
6 & 0.00457 & 0.00413 & 0.00314 & 0.00205 & 0.00113 & 0.00045 \\
\hline \hline
\end{tabular}

Table 2: Values of $\beta$ for the first pairs of $\left(n_{x}, n_{y}\right)$.

\begin{tabular}{ccccccc}
\hline \hline$\frac{n_{y}}{n_{y}}$ & 0 & 1 & 2 & 3 & 4 & 5 \\
\hline \hline 0 & 0.18169 & 0.04507 & 0.01315 & 0.00469 & 0.00205 & 0.00106 \\
1 & 0.04507 & 0.03052 & 0.01596 & 0.00826 & 0.00451 & 0.00264 \\
2 & 0.01315 & 0.01596 & 0.01192 & 0.00789 & 0.00510 & 0.00334 \\
3 & 0.00469 & 0.00826 & 0.00789 & 0.00626 & 0.00464 & 0.00337 \\
4 & 0.00205 & 0.00451 & 0.00510 & 0.00464 & 0.00384 & 0.00304 \\
5 & 0.00106 & 0.00264 & 0.00334 & 0.00337 & 0.00304 & 0.00259 \\
6 & 0.00062 & 0.00165 & 0.00225 & 0.00244 & 0.00236 & 0.00214 \\
\hline \hline
\end{tabular}

Table 3: Values of $\alpha$ 's on the plane $z=0$, for pairs of $\left(n_{x}, n_{y}\right)$.

\begin{tabular}{ccccccc}
\hline \hline$\frac{n_{x}}{n_{y}}$ & 0 & 1 & 2 & 3 & 4 & 5 \\
\hline \hline 0 & 0.33333 & 0.06175 & 0.01392 & 0.00404 & 0.00153 & 0.00073 \\
1 & 0.06175 & 0.02323 & 0.00793 & 0.00298 & 0.00130 & 0.00066 \\
2 & 0.01392 & 0.00793 & 0.00379 & 0.00184 & 0.00095 & 0.00054 \\
3 & 0.00404 & 0.00298 & 0.00184 & 0.00109 & 0.00066 & 0.00041 \\
4 & 0.00153 & 0.00130 & 0.00095 & 0.00066 & 0.00045 & 0.00031 \\
5 & 0.00073 & 0.00066 & 0.00054 & 0.00041 & 0.00031 & 0.00023 \\
6 & 0.00040 & 0.00038 & 0.00033 & 0.00027 & 0.00022 & 0.00017 \\
\hline \hline
\end{tabular}

\section{References}

[1] H. Takayasu, Phys. Rev. Lett. 54, 1099 (1985).

[2] J.C. Dyre. and Th. Schroder B, Rev. of Modern Phys.
72, $873(2000)$

[3] A. Hansen, E.L. Hinrichsen and S. Roux, Phys. Rev. B 43, 665 (1991) and in Phys. Rev. Lett. 66, 2476 (1991). 
[4] V.K.S. Shante and S. Kirkpatric, Adv. Phys. 20, 325 (1971).

[5] M. Sahimi and J.D. Goddard, Phys. Rev. B 32, 1869 (1985)

[6] J.W. Chung, A. Ross, J. Th. De Hosson and E van der Giessen, Phys. Rev. B 54, 15094 (1996-I).

[7] P. Ray and B.K. Chakrabarti, Phys. Rev. B 38, 715 (1988).

[8] Y. Kantor and I. Webman, Phys. Rev. Lett. 52, 1891 (1984)

[9] H.J. Herrmann, A. Hansen and S. Roux, Phys. Rev. B39, 637 (1989).

[10] B.K. Chakrabarti and L.G. Benguigui, "Statistical Physics of Fracture and Breakdown ind Disordered Systems”, Oxford University Press, Inc. N. Y., 1997.
[11] P.M. Duxbury, P.D. Beale and P.L. Leath, Phys. Rev. Lett 57,1052(1986) and in Phys. Rev. B 36, 367 (1986).

[12] S. Kirkpatric, Rev. of Modern Phys. 45, 574 (1973).

[13] J. Bernasconi, Phys. Rev. B 9, 4575 (1974).

[14] D.G. Harlow and S.L. Phoenix, Int. J. Frac. 17, 601 (1981).

[15] J.S.Espinoza

Ortiz,Chamith S. Rajapakse, and Gemunu H.Gunaratne, Phys. Rev. B. 66, 144203 (2002) and in Virtual Journal of Biological Physics Research-Multicellular Phenomena 4, issue 9, (2002).

[16] G.H. Gunaratne, C.S. Rajapakse, K.E. Bassler, K.K. Mohanty and S.J. Wimalawansa, Phys. Rev. Lett. 880, 68101 (2002). 